\title{
Text messaging reminders to reduce non-attendance in chronic disease follow-up: a clinical trial
}

\author{
Su-May Liew, Seng Fah Tong, Verna Kar Mun Lee, Chirk Jenn Ng, Kwok Chi Leong and Cheong Lieng Teng
}

\section{ABSTRACT}

\section{Background}

Non-attendance results in administrative problems and disruption in patient care. Several interventions have been used to reduce non-attendance, with varying degree of success. A relatively new intervention, text messaging, has been shown to be as effective as telephone reminders in reducing non-attendance. However, no study has looked specifically at using text messaging reminders to reduce non-attendance in chronic disease care.

Aim

To determine if text messaging would be effective in reducing non-attendance in patients on long-term followup, compared with telephone reminders and no reminder Design of study

A randomised controlled trial with three arms: text messaging reminder, telephone reminder, and control. Setting

Two primary care clinics in Malaysia.

Method

A total of 931 subjects who had been on at least 6 months of follow-up were randomised into the three groups. Demographic variables were recorded at the first visit. In the intervention arms, a reminder was sent 24-48 hours prior to the appointment. Non-attendance rate was documented at the second visit. Non-attenders were defined as those who did not attend, attended early, or attended late without rescheduling their appointment. Attenders were defined as participants who had turned up for their scheduled appointment and those who had changed or cancelled their appointment with notification.

\section{Results}

The non-attendance rates in the text messaging group (odds ratio $[\mathrm{OR}]=0.62,95 \%$ confidence interval $[\mathrm{Cl}]=$ 0.41 to $0.93, P=0.020$ ) and the telephone reminder group (OR $=0.53,95 \% \mathrm{Cl}=0.35$ to 0.81$), P=0.003$ ) were significantly lower than the control group. The absolute non-attendance rate for telephone reminders was lower by $2 \%$ compared to the text messaging group. This difference was not found to be statistically significant $(P=0.505)$.

\section{Conclusion}

Text messaging was found to be as effective as telephone reminder in reducing non-attendance in patients who required long-term follow-up for their chronic illnesses in this study. It could be used as an alternative to conventional reminder systems.

\section{Keywords}

non attendance; primary health care; randomised controlled trial (RCT); reminder systems; cellular phone.

\section{INTRODUCTION}

Non-attendance is a huge health administration and economic problem. It has been estimated that $£ 150$ million is lost each year because of the failure of patients to keep appointments with their GPs in the UK. ${ }^{1}$ When patients default clinic appointments, it causes disruption in patient care; the waiting times are longer, not only for themselves but also for other patients. ${ }^{1,2}$ Several studies have investigated the causes of non-attendance,,$^{3-6}$ as well as evaluating interventions to reduce non-attendance. These interventions include telephone reminders, orientation statements, and postal reminders. ${ }^{7,8}$ Telephone and postal reminders have been shown to reduce non-attendance, but these interventions have their limitations. One study showed that the effect of mailed reminders decreases with time. ${ }^{9}$ Telephone reminders are costly and repeated calls may be needed to make a single contact. Orientation statements provide patients with information about

SM Liew, MBBS, MMed(Fam Med), senior lecturer; CJ Ng, MBBS, MMed(Fam Med), associate professor, Department of Primary Care Medicine, University of Malaya; SF Tong, $M B B S, M M e d(F a m$ Med), senior lecturer, Universiti Kebangsaan Malaysia; VKM Lee, MD, MMed(Fam Med) senior lecturer; CL Teng, MBBS, MMed(Fam Med), professor, Department of Family Medicine, International Medical University, Malaysia. KC Leong; FRACGP, clinical tutor, School of Medicine, Deakin University, Geelong, Victoria, Australia.

Address for correspondence

Dr Su-May Liew, University of Malaya, Department of Primary Care Medicine, Faculty of Medicine, Lembah Pantai, Kuala Lumpur, 50603, Malaysia. E-mail: sumayliew@gmail.com

Submitted: 16 September 2008; Editor's response: 11 December 2008; final acceptance: 18 March 2009.

(c) British Journal of General Practice.

This article was originally online first on 26 Aug 2009. Cite this article as: Br J Gen Pract 2009; 59: 916-920. Advance online publication. DOI: 10.3399/bjgp09X472250. 
the reason for the appointment and clinic organisation. This intervention has had varied results, with one study showing that it was not effective when tested in medical patients. ${ }^{7}$

Text messaging, a newer telecommunication technology, offers an alternative strategy to the conventional reminder systems. ${ }^{10-12}$ Leong et al showed that both telephone reminders and text messaging reminders significantly reduced nonattendance rates in seven primary care clinics, when compared to no reminder. ${ }^{13}$ The study also showed that using text messaging reminders was more costeffective than telephone reminders. The service has been proposed as being particularly useful in patients with chronic disease, ${ }^{13}$ where appointments are scheduled every 3 to 6 months and patients are given the next appointment at the end of their visit. Moreover, non-attendance in this group might also result in more adverse health outcome.

Text messaging is noted for its speed and accessibility. ${ }^{14} \quad$ Banking, ${ }^{15}$ advertising, ${ }^{16}$ and entertainment ${ }^{14}$ industries have already utilised text messaging to reach out to consumers. The healthcare system is beginning to test and apply this technology in different ways in order to improve patient care. ${ }^{17-19}$ In a qualitative study looking at asthma outpatient non-attendance, patients spontaneously brought up the subject of text messaging as a reminder of future appointments. ${ }^{3}$

The present study is important because it attempted to investigate the feasibility of using a new innovation in health service delivery to reduce nonattendance. Text messaging could potentially reduce costs and improve patient care by specifically targeting a population that would benefit most from attending follow-up.

It was hypothesised that text messaging reminder was more effective than no reminder (control) and was as effective as telephone reminder in reducing non-attendance in patients with chronic diseases.

\section{METHOD}

\section{Population and setting}

This was a randomised controlled trial. Two primary care clinics in Kuala Lumpur, Malaysia that catered for patients requiring chronic disease care were chosen. Both clinics are affiliated to teaching hospitals: one is based in the grounds of the hospital itself, while the other is in a residential housing area. The clinics are located in an urban setting with a similar practice profile consisting of patients who have chronic diseases such as diabetes, asthma, hypertension, dyslipidaemia, and coronary artery disease. Each clinic had an average of two academic staff with a number of postgraduate trainees in family medicine and medical officers. The inclusion criteria

\section{How this fits in}

Text messaging has previously been shown to reduce non-attendance and to

be more cost-effective than conventional reminders such as telephone

reminders in various clinic settings. This study looked specifically at patients on

long-term follow-up and it showed that text messaging is as effective as

telephone reminder in improving non-attendance in patients receiving care for

their chronic illnesses. As management of chronic disease in primary care is a

lifelong process, text messaging reminder should be considered an alternative

means to improve the health care of these patients.

were that the participants must have: (a) registered with the clinics for at least 6 months; (b) at least one chronic disease; (c) a return appointment between 1 and 6 months; and (d) ownership of a mobile phone by the patient or an accompanying person who would be able to contact the patient. Exclusion criteria were: illiteracy or inability to read or understand text messaging due to medical conditions such as cognitive and visual impairment.

\section{Sample size}

The participants were randomised into three groups: text messaging reminder, telephone reminder, and no reminder (control group). A nonattendance rate of $30 \%$ was expected in the control group and $20 \%$ in the telephone reminder group. In order to detect a $15 \%$ absolute difference in nonattendance rate between text messaging reminder and the control group, 121 patients were needed in each of the three arms (power $80 \%, \alpha=5 \%$ ); for a $10 \%$ absolute difference in non-attendance rate between text messaging reminder and telephone reminder, 294 patients were needed in each arm. The latter figure was used to calculate the sample size for this study.

\section{Randomisation and intervention}

Randomisation was by computer-generated block randomisation using a block size of three units. It was estimated that 300 participants in each arm and 450 participants per centre were needed. Patients were recruited if they fulfilled the inclusion criteria. Written consent was obtained, and the participants were then given an anonymous identification code number according to the time of entry into the study. The recruiters who enrolled the research subjects were blind to the intervention at the time of recruitment. Assignment of participants to the three groups was by computer allocation using the list of anonymous identification codes.

Reminders (or no reminder) were sent to the participants 24-48 hours before the scheduled appointment. To avoid caller bias during telephone conversations, a research assistant was trained to 
Figure 1. Participant flow.

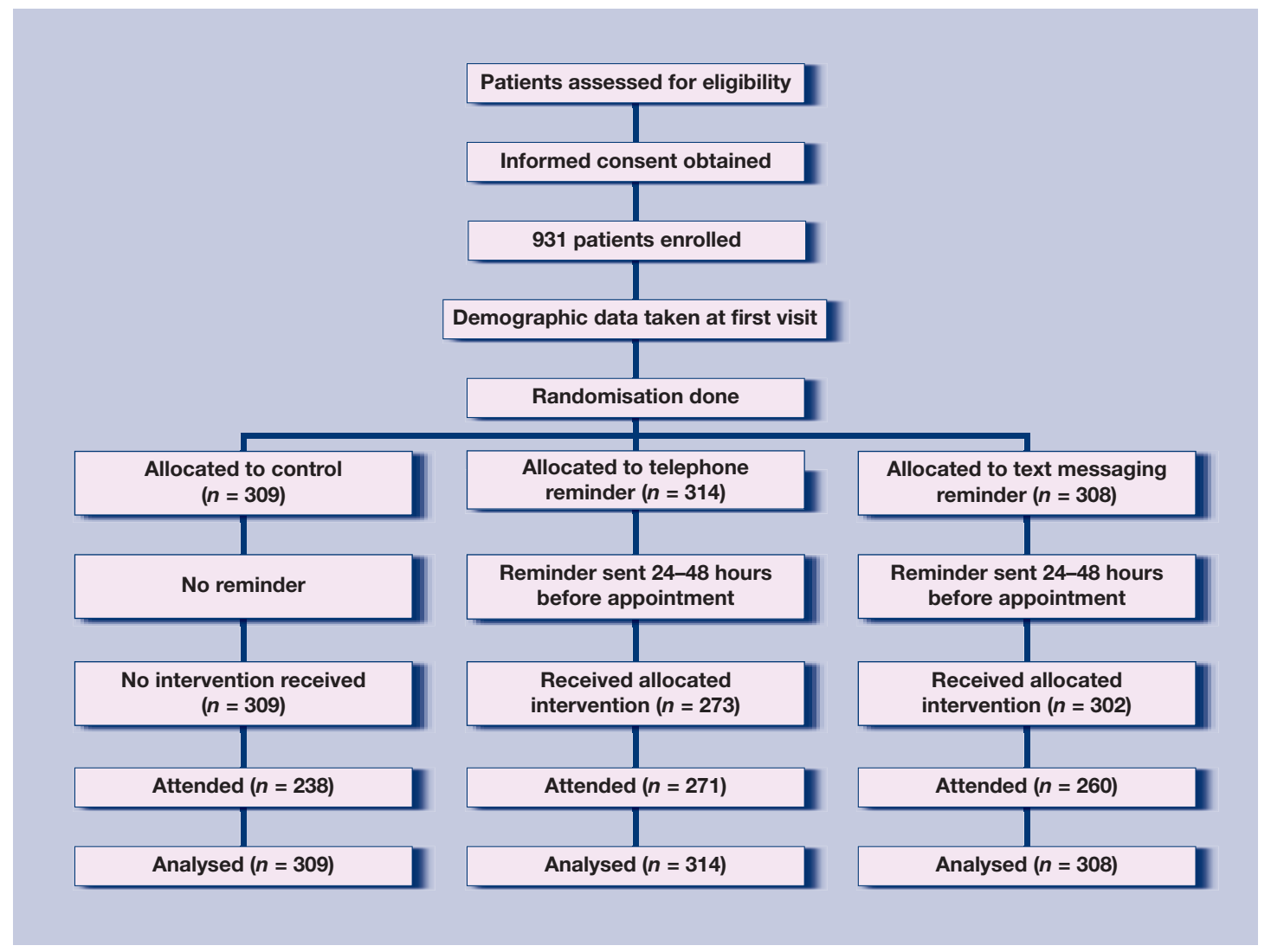

deliver the same telephone message as in the telephone reminder group. Any further enquiries from the patient were redirected to the appointment counter. If the contact was unsuccessful, up to three further attempts were made at 4-hourly intervals. Similarly, a standard text message was used for the text messaging reminder group. Intervention was considered to have been received if participants answered the phone in the telephone reminder group or when 'message sent' was recorded on the mobile phone in the text messaging reminder group. Patients' demographic data were gathered at the first visit and their attendance documented at the scheduled follow-up visit.

Table 1. Demographic data of study population.

\begin{tabular}{lccc} 
& $\begin{array}{c}\text { Control } \\
n=309\end{array}$ & $\begin{array}{c}\text { Telephone reminder, } \\
n=314\end{array}$ & $\begin{array}{c}\text { Text messaging } \\
\text { reminder, } n=308\end{array}$ \\
\hline Mean age, years $^{\mathrm{a}}$ & 60.77 & 57.73 & 58.19 \\
\hline Female sex (\%) & $173(56.0)$ & $190(60.5)$ & $168(54.5)$ \\
\hline $\begin{array}{l}\text { Mobile phone owner } \\
\text { Patient }\end{array}$ & & & \\
$\quad$ Relative or friend & $241(78.0)$ & $211(67.2)$ & $211(68.5)$ \\
\hline Chronic disease, $n(\%)$ & $68(22.0)$ & $103(32.8)$ & $97(31.5)$ \\
$\quad \begin{array}{lll}\text { Diabetes } \\
\text { Hypertension }\end{array}$ & $130(25.6)$ & $153(29.3)$ & $155(30.6)$ \\
Hyperlipidaemia & $239(47.0)$ & $233(44.6)$ & $200(43.6)$ \\
\hline
\end{tabular}

${ }^{\mathrm{a}} d f=2, F=4.44, \mathrm{P}=0.012 .{ }^{\circ} d f=2, \chi^{2}=10.5, \mathrm{P}=0.005$.

\section{Outcome measurement and statistical} analysis

Attenders were defined as participants who had turned up for their scheduled appointment and those who had changed or cancelled their appointment with notification. Non-attenders were those who did not attend, attended early, or attended late without rescheduling their appointment. SPSS (version 11.5) was used for data analysis. The attendance rates among the intervention groups were calculated based on intention-to-treat principle. The $\chi^{2}$ test was used to compare the categorical variable (nonattendance versus attendance in clinic). The level of statistical significance was set at 0.05 . For odds ratios (OR) calculation (and 95\% confidence interval $[\mathrm{CI}])$, the attendance rate of the no reminder group was the reference group.

\section{RESULTS}

A total of 931 patients were recruited into the study (Figure 1). Demographic data of the subjects are shown in Table 1. The frequency of the chronic diseases was similar in all three groups. Hypertension was the most frequent chronic illness among the patients in this study. However, there were more patients who owned a mobile telephone in the control group $(78.0 \%)$ compared to the intervention groups (telephone $67.2 \%$ and text messaging $68.5 \% ; \chi^{2}=$ 10.5 , degrees of freedom (df) $=2, P=0.005$ ). 
The non-attendance rates are summarised in Table 2.

When compared to control, the non-attendance rate was significantly lower in the text messaging and telephone reminder groups. However, there was no statistical difference between the two reminder groups $(P=0.505)$.

In the telephone reminder group, the number of attempts required for each successful reminder to be received was recorded; $83 \%$ required only one phone call, 9\% required two phone calls, and $8 \%$ required three phone calls (data available from 309 participants).

\section{DISCUSSION}

\section{Summary of main findings}

The results of this study showed that both text messaging and telephone reminders were effective in reducing non-attendance in people who required longterm follow-up for chronic diseases. The reduction in non-attendance by the two reminder methods demonstrates that forgetfulness is a major reason for defaulting follow-up in chronic disease care.

It was also hypothesised that text messaging was as effective as telephone reminder, and analysis showed that there was no significant difference in non-attendance rates between the two reminder groups. It is possible that there exists a real difference with telephone reminder being more effective than text messaging and that this is less than the $10 \%$ difference that was used to calculate sample size. However, it is unlikely that this is clinically important.

The advantages of text messaging, such as speed, convenience, ${ }^{14}$ and cost-effectiveness, ${ }^{13}$ are likely to outweigh the minimal increase in attendance by telephone reminders. A possible reason why telephone reminders were slightly more effective in this study is that the participants were older, with a mean age of 58.7 years. In banking, it was found that older consumers, unlike the younger ones, regarded face-to-face contact to be more important than convenience. They were also less likely to accept newer technologies like mobile banking. ${ }^{15}$

\section{Strengths and limitations of the study}

A limitation of this study was that the costeffectiveness of the two reminder methods was not studied. Text messaging is cheaper than telephone calls with regard to telephone company fees. However, the greater cost is the time spent by staff in making telephone calls as compared to sending prerecorded text messages. Twenty per cent of participants in the telephone reminder group required more than one attempt for successful delivery of the reminder.

\begin{tabular}{l} 
Table 2. Non-attendance rates in control, telephone and \\
text-messaging groups. \\
\cline { 2 - 6 } \\
\begin{tabular}{lcccc}
$n$ & $\%$ & $P$-value & Odds ratio $(95 \% \mathrm{Cl})$ \\
\hline Control & 314 & 13.7 & 0.003 & $0.53(0.35$ to 0.81$)$ \\
\hline Telephone reminder & 308 & 15.6 & 0.020 & $0.62(0.41$ to 0.93$)$ \\
\hline Text messaging reminder & & & &
\end{tabular}
\end{tabular}

This study aimed to detect a $15 \%$ difference in non-attendance rates between the telephone and text messaging reminder groups and the control group. However, the differences in non-attendance rates between telephone and control groups (9.3\%, $P=0.003)$ as well as between text messaging and control groups $(7.4 \%, P=0.020)$ were smaller than expected. The statistically significant difference was due to the larger sample size, which was calculated with the aim to detect a $10 \%$ difference in nonattendance rates between text messaging and telephone reminders. Therefore, the impact of telephone or text messaging reminders may have been over-estimated.

Furthermore, the baseline (control) nonattendance rate (23\%) was also lower than expected, thus contributing to greater difficulty in producing a large reduction in non-attendance. It should be noted that in another recent randomised control trial of telephone and text messaging reminders in a clinic in China, Chen et al demonstrated a reduction of nonattendance in the order of $7-8 \%$ only. ${ }^{20}$ This randomised controlled trial specifically addressed the efficacy of reminder systems on patients with long-term follow-up. The subject matter would be of interest to all organisations that have clients making appointments.

\section{Comparison with existing literature}

The present results are similar to those of another study conducted in seven primary care clinics in Kuala Lumpur where text messaging was found to be as effective as telephone reminders in significantly reducing non-attendance. ${ }^{13}$ The attendance rates for telephone reminders $(59.6 \%$; OR $=1.55)$ and text messaging (59.0; OR = 1.59) were similar. The mean age of their patient population was much lower at 38.2 years.

Chen et al conducted a randomised controlled trial on reminders in a health promotion clinic and again the results showed higher attendance rates for telephone reminders $(88.3 \%$; OR $=1.83)$ and text messaging reminders $(87.5 \%$; OR $=1.70)$ when compared to control. ${ }^{20}$ The attendance rate in their control group was high at $80.5 \%$ compared to the present study and the study carried out by Leong et al. ${ }^{13}$

Two studies by Downer et al showed text messaging to be effective in lowering non- 
attendance rates, but both were conducted in a children and adolescent hospital. ${ }^{11,19}$ The efficacy of text messaging may differ according to the age of the population of the clinic being studied, and this should be investigated in future research.

Fairhurst and Sheikh focused their study on repeated non-attenders in a general practice in Scotland. ${ }^{21}$ In their study, only patients who failed to attend two or more appointments in the preceding 12 months were included. For this group of patients, text messaging showed only a $5 \%$ absolute reduction in non-attendance when text messaging was compared to a control group, and the difference was not significant. It is possible that in this group of patients, interventions, other than appointment reminders, are required.

\section{Implications for future research and clinical practice}

In order to improve health service delivery, interventions to reduce non-attendance should be shown to be effective and economical. The impact of improved attendance would be most felt in patients receiving long-term follow-up where disruption in patient care is deleterious. This study showed text messaging to be effective in reducing nonattendance in patients with chronic disease on longterm care.

Further research needs to look at different populations and settings. It is possible that the use of more than one text message reminder, or combinations of reminders, may be more effective, and this may be done through an automated reminder system.

\section{Funding body}

This study was made possible with research funding from University of Malaya (reference F0381/2005C) and University Kebangsaan Malaysia (reference FF-225-2005)

\section{Ethics committee}

Medical ethics committee - University of Malaya Medical Centre (reference 454.3) and medical ethics committee Universiti Kebangsaan Malaysia (reference FF-225-2005)

\section{Competing interests}

The authors have stated that there are none

\section{Acknowledgements}

We are indebted to our patients for their participations in the study and to the research assistants for their help in patient recruitment to the study.

\section{Discuss this article}

Contribute and read comments about this article on the Discussion Forum: http://www.rcgp.org.uk/bjgp-discuss

\section{REFERENCES}

1. Beecham L. Missed GP appointments cost NHS money. BMJ 1999; 319(7209): 536.

2. Griffin SJ. Lost to follow-up: the problem of defaulters from diabetes clinics. Diabet Med 1998; 15(suppl 3): S14-S24.

3. Van Baar, Joosten H, Car J, et al. Understanding reasons for asthma outpatient (non)-attendance and exploring the role of telephone and e-consulting in facilitating access to care: exploratory qualitative study. Qual Saf Health Care 2006; 15(3): 191-195.

4. Neal RD, Gambles MH, Allgar VL, et al. Reasons for and consequences of missed appointments in general practice in the UK: questionnaire survey and prospective review of medical records. BMC Fam Pract 2005; 6: 47

5. Martin C, Perfect T, Mantle G. Non-attendance in primary care: the views of patients and practices on its causes, impact and solutions. Fam Pract 2005; 22(6): 638-643.

6. Zailinawati $\mathrm{AH}, \mathrm{Ng} \mathrm{CJ}$, Nik-Sherina $\mathrm{H}$. Why do patients with chronic illnesses fail to keep their appointments? A telephone interview. Asia Pac J Public Health 2006; 18(1): 10-15.

7. Macharia WM, Leon G, Rowe BH, et al. An overview of interventions to improve compliance with appointment keeping for medical services. JAMA 1992; 267(13): 1813-1817.

8. Sawyer SM, Zalan A, Bond LM. Telephone reminders improve adolescent clinic attendance: a randomized controlled trial. $J$ Paediatr Child Health 2002; 38(1): 79-83.

9. Morse DL, Coulter MP, Nazarian LF, Napodano RJ. Waning effectiveness of mailed reminders on reducing broken appointments. Pediatrics 1981; 68(6): 846-849.

10. Castano P, Andres R, Lara M, Westhoff C. Assessing feasibility of text messaging to improve medication adherence. Obstet Gynecol 2006; 107(4): 40S.

11. Downer S, Meara JG, Da Costa AC. Use of text messaging to improve outpatient attendance. Med J Australia 2005; 183(7): 366-368.

12. Patel A, Howard J, Anie K. The use of a text messaging service to improve attendance at a sickle cell clinic (poster presentations: red cell disorders). Br J Haematol 2007; 137(suppl 1): 31.

13. Leong KC, Chen WS, Leong KW, et al. The use of text messaging to improve attendance in primary care: a randomized controlled trial. Fam Pract 2006; 23(6): 699-705

14. Tsai M. Telecommunications. What's a phone for? In Asia, probably a lot more than you can imagine. Wall St J (East Ed) 2006; June 19: R12.

15. Howcroft B, Hamilton R, Hewer P. Consumer attitude and the usage and adoption of home-based banking in the United Kingdom. Int J Bank Mark 2002; 20(3): 111-121.

16. Rettie R, Grandcolas U, Deakins B. Text message advertising: response rates and branding effects. Journal of Targeting, Measurement and Analysis for Marketing 2005; 13(4): 304-312.

17. Malik A, Saeed A, Bradbury S, Bullimore DW. Towards a better outpatient service in inflammatory bowel disease. Gut 2007; 56(suppl 2): A17.

18. Menon-Johansson AS, McNaught F, Mandalia S, Sullivan AK. Texting decreases the time to treatment for genital Chlamydia trachomatis infection. Sex Transm Infect 2007; 82(1): 49-51.

19. Downer SR, Meara JG, Da Costa AC, Sethuraman K. SMS text messaging improves outpatient attendance. Aust Health Rev 2006; 30(3): 389-396.

20. Chen ZW, Fang LZ, Chen LY, Dai HL. Comparison of an SMS text messaging and phone reminder to improve attendance at a health promotion center: a randomized controlled trial. J Zhejiang Univ Sci B 2008; 9(1): 34-38.

21. Fairhurst K, Sheikh A. Texting appointment reminders to repeated non-attenders in primary care: randomised controlled study. Qual Saf Health Care 2008; 17: 373-376. 